\title{
PLANEJANDO O PROCESSO DE PREVISÃO DE DEMANDA COM AUXÍLIO DA LÓGICA FUZZY
}

\section{P.Senna $^{1^{*}}$, R. Tanscheit ${ }^{2}$ e A. M. Gomes ${ }^{1}$}

1 Centro Federal de Educação Tecnológica Celso Suckow da Fonseca, 26041-271, Nova Iguaçu-RJ, Brasil

2 Pontifícia Universidade Católica do Rio de Janeiro, PUC-RIO, 22451-900, Rio de Janeiro-RJ

\author{
* pedro.sennavieira@gmail.com
}

\section{RESUMO}

De uma forma ampla, prever a demanda de forma acurada é parte fundamental do processo de Gestão de Demanda das empresas. Como em muitos casos não é possível que a produção seja sob encomenda, há a necessidade de estimar a quantidade de produtos a serem vendidos e utilizar esta previsão. Considerando este cenário, prever demanda com o mínimo erro possível deve ser a principal prioridade para as empresas. Para atingir este objetivo, são apresentadas, basicamente, duas técnicas de previsão: a Decomposição Clássica e a previsão baseada em Lógica Fuzzy. Como principal resultado, pode-se ressaltar o baixo erro encontrado por ambas as técnicas, com pequena vantagem para a Lógica Fuzzy.

PALAVRAS-CHAVE: Previsão de Demanda, Decomposição Clássica, Lógica Fuzzy.

\section{DEMAND FORECAST PROCESS PLANNING WITH FUZZY LOGIC SUPPORT}

\begin{abstract}
Generally, demand forecast is a crucial part of demand management process. As in many cases production cannot be of the make-to order type, it becomes necessary how many products will be sold. In this scenario, minimizing sales forecasting error should be the top priority in any company. In order to attain this
\end{abstract}

goal, two techniques are presented here: Classic Decomposition and Forecast based on Fuzzy Logic. The main result is the low error found when applying both techniques, with a slight advantage for the latter.

KEYWORDS: Demand Forecasting, Classic Decomposition, Fuzzy Logic. 


\section{INTRODUÇÃO}

Prever a demanda é uma ação muito importante e presente na rotina de pequenas, médias ou grandes empresas, seja de maneira mais intuitiva e rudimentar (simplesmente repetindo as vendas do período anterior) ou utilizando métodos estatísticos mais rebuscados (análise de dados históricos, por exemplo). Todo negócio desenvolve em algum momento um modelo de previsão de demanda, que é capaz de auxiliar a tomada de decisões no ambiente empresarial.

Entende-se por demanda a disposição dos clientes ao consumo de bens ou serviços ofertados por uma empresa. Esta demanda pode ser influenciada por diversos fatores, desde as condições macroeconômicas até questões operacionais, como a disponibilidade de matéria-prima para iniciar o processo produtivo ou o preço no ponto de venda. Por este motivo, no processo de previsão de demanda, é comum rever os dados referentes aos períodos de vendas atípicas.

Neste trabalho busca-se efetuar a previsão de demanda com base em dados históricos a partir de dois métodos. Um é amplamente divulgado em escolas de Administração: denomina-se Decomposição Clássica e permite a projeção de tendências com sazonalidade. O outro, não tão difundido nesse meio, consiste em prever a demanda utilizando a Lógica Fuzzy, que pode ser interpretada como um processo de composição de relações fuzzy.

Este artigo está estruturado em cinco seções, com os conteúdos apresentados na sequência. Na seção 1 são apresentados, o problema e os objetivos principais. Na seção 2 são apresentados os materiais e métodos de pesquisa aplicados neste trabalho. A seção 3 trata dos fundamentos conceituais, isto é, as principais referências bibliográficas que embasaram este trabalho. A seção 4 aborda a análise e discussão dos resultados. A seção 5 apresenta as conclusões e estudos futuros.

\section{MATERIAIS E MÉTODOS}

A Erro! Fonte de referência não encontrada. mostra as macro etapas do trabalho com base na metodologia de estudo de caso de Yin (2010).

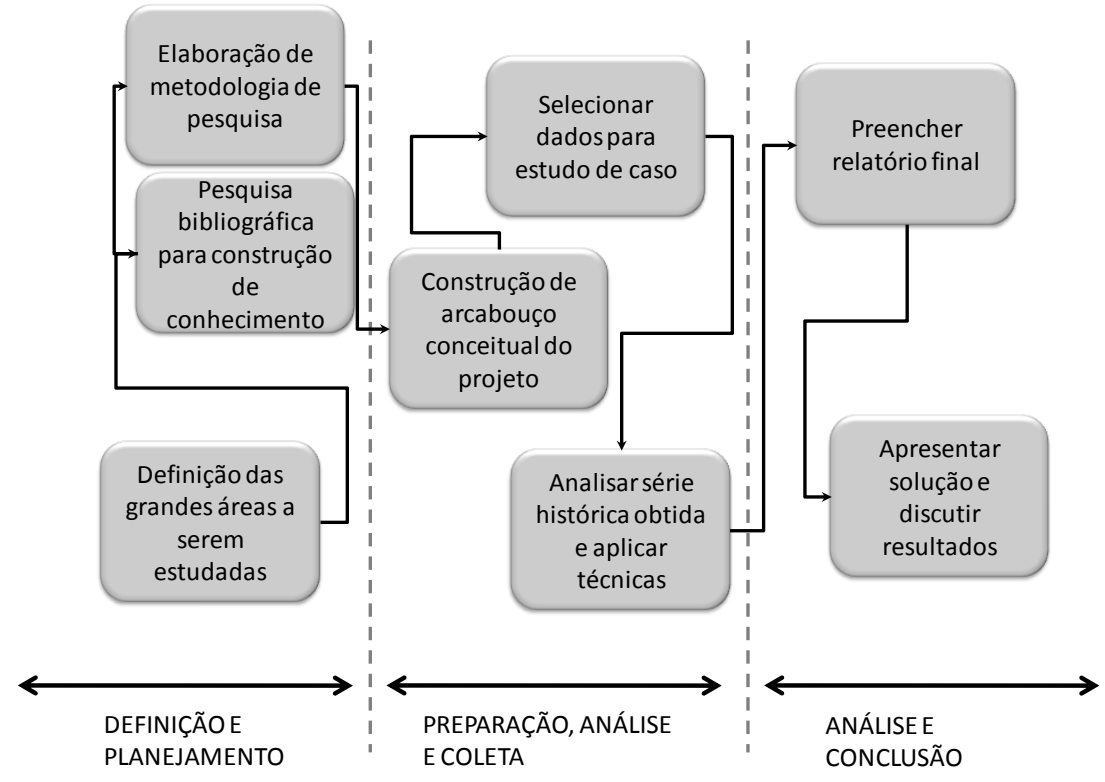

Figura 1- Metodologia de Pesquisa

Fonte: Elaborado pelos autores (2015)

As etapas apresentam as seguintes características: 
a) Definição das grandes áreas a serem estudadas: crucial para que se estabeleça o escopo de atuação do projeto e para que o trabalho fique completo conceitualmente, sem a preocupação, no entanto, de abordar todos os conceitos relacionados com o tema, mas sim de apresentar uma contribuição à discussão proposta.

b) Pesquisa bibliográfica para construção de conhecimento: uma vez estabelecidos os campos do conhecimento, efetua-se a busca bibliográfica para adquirir conhecimento a respeito dos temas.

c) Elaboração da metodologia de pesquisa: fundamental para garantir rigor científico ao trabalho. A boa descrição destas etapas servirá de auxílio a quem desejar seguir a mesma linha de pesquisa.

d) Construção do arcabouço conceitual do projeto: esta etapa é cumprida a partir da pesquisa e leitura de teses, artigos e periódicos relacionados ao tema do trabalho. Desta forma é construído o corpo de conhecimentos que serve de base para a abordagem do problema proposto.

e) Selecionar dados para estudo de caso: etapa que costuma ser a mais crítica em um projeto deste porte. Apesar de serem claros os benefícios para as empresas (recebem uma solução para um problema real a custo zero), estas relutam muito em divulgar seus dados operacionais (ainda que alterados e com o nome da empresa em sigilo). Tal fato torna difícil a obtenção de dados reais para o caso prático. Neste caso, a solução encontrada foi utilizar séries históricas disponíveis em FIRJAN (2011). A série histórica escolhida para efetuar a previsão foi a série de vendas têxteis. Apesar de ser um agregado do mercado como um todo, este é um dado interessante para que as empresas possam ter noção do mercado que há para ser explorado.

f) Analisar série histórica obtida e aplicar técnicas: uma vez obtida a série histórica, aplicam-se as técnicas pertinentes ao escopo do trabalho e comparações são realizadas.

g) Preencher relatório final: esta etapa consiste na elaboração deste trabalho, compilando todos os experimentos realizados, as dificuldades encontradas, bibliografias pesquisadas e sugerindo trabalhos futuros.

h) Apresentar solução e discutir resultados: uma vez desenvolvido o trabalho, inicia-se a discussão sobre eventuais aprimoramentos e desdobramentos. Como melhorar os resultados encontrados? Como criticá-los de forma construtiva? $O$ que pode ser aprimorado para que o trabalho adquira maior robustez e contribua mais para a sociedade? Esta etapa engloba este debate amplo.

\section{REVISÃO DE LITERATURA}

Para embasar o trabalho tecnicamente, faz-se necessário revisar parte da literatura qualificada acerca do tema. Na primeira parte desta seção efetua-se uma discussão conceitual da Gestão de Demanda e da importância da previsão da demanda dentro deste conceito. Na segunda parte, abordam-se a Decomposição Clássica e a Lógica Fuzzy, tendo em vista o problema de previsão $d$ a demanda.

Uma busca pelo termo "fuzzy" na base SCOPUS produz mais de 500.000 resultados. O gráfico da Figura 2 mostra a evolução das publicações ao longo do tempo. Mais especificamente, ressalta-se um crescimento mais acentuado do número de publicações a partir da segunda metade dos anos 90, o que é explicado pela maior popularização dos computadores. 


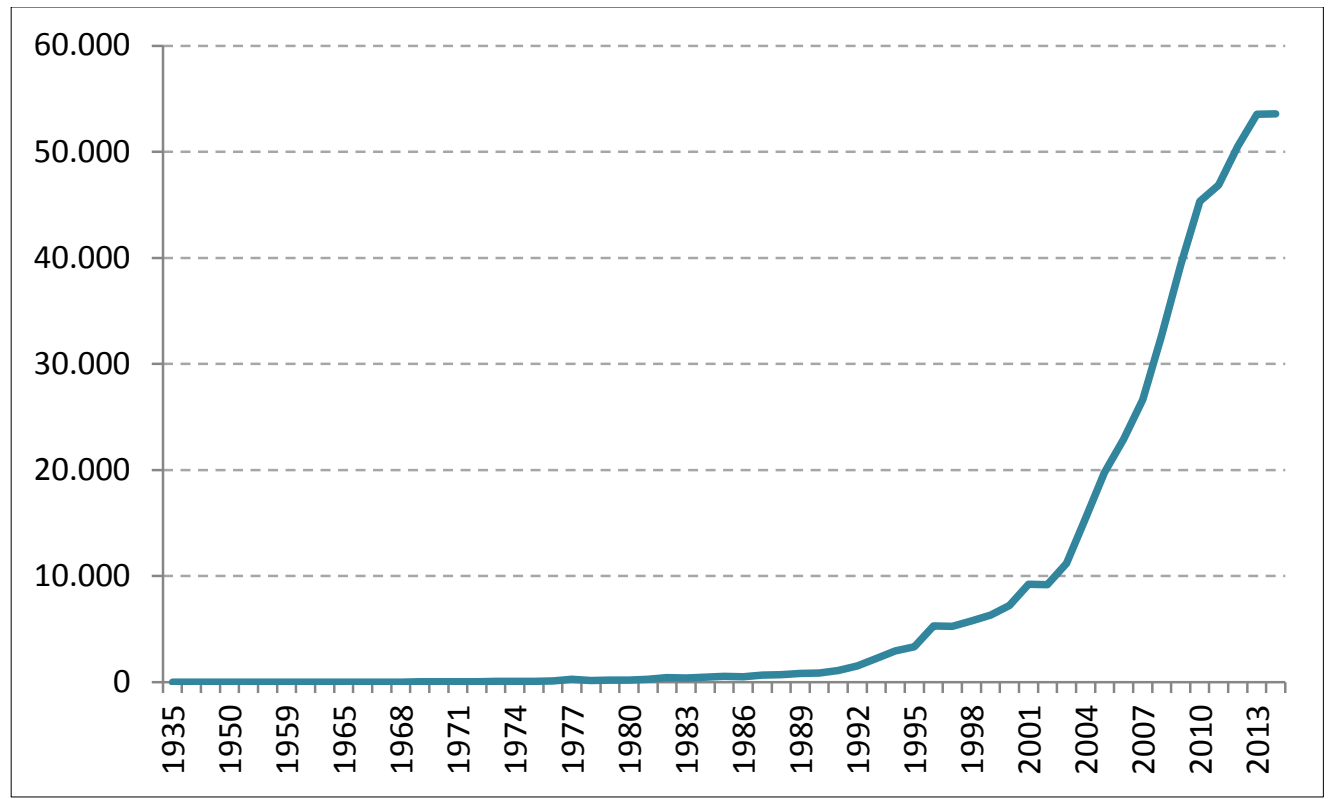

Figura 2- Quantidade de publicações onde o termo "fuzzy" é encontrado na Scopus

Fonte: Autores (2015)

No entanto, efetuando uma busca pelo termo "sales forecast" dentro deste universo de 500.000 publicações, são retornadas apenas 99 ocorrências, o que mostra que ainda há muito potencial de pesquisa no que diz respeito à aplicação da Lógica Fuzzy em previsão de vendas. A Figura 3 mostra o aumento da relevância também para a combinação dos termos "fuzzy" e "Sales forecast"

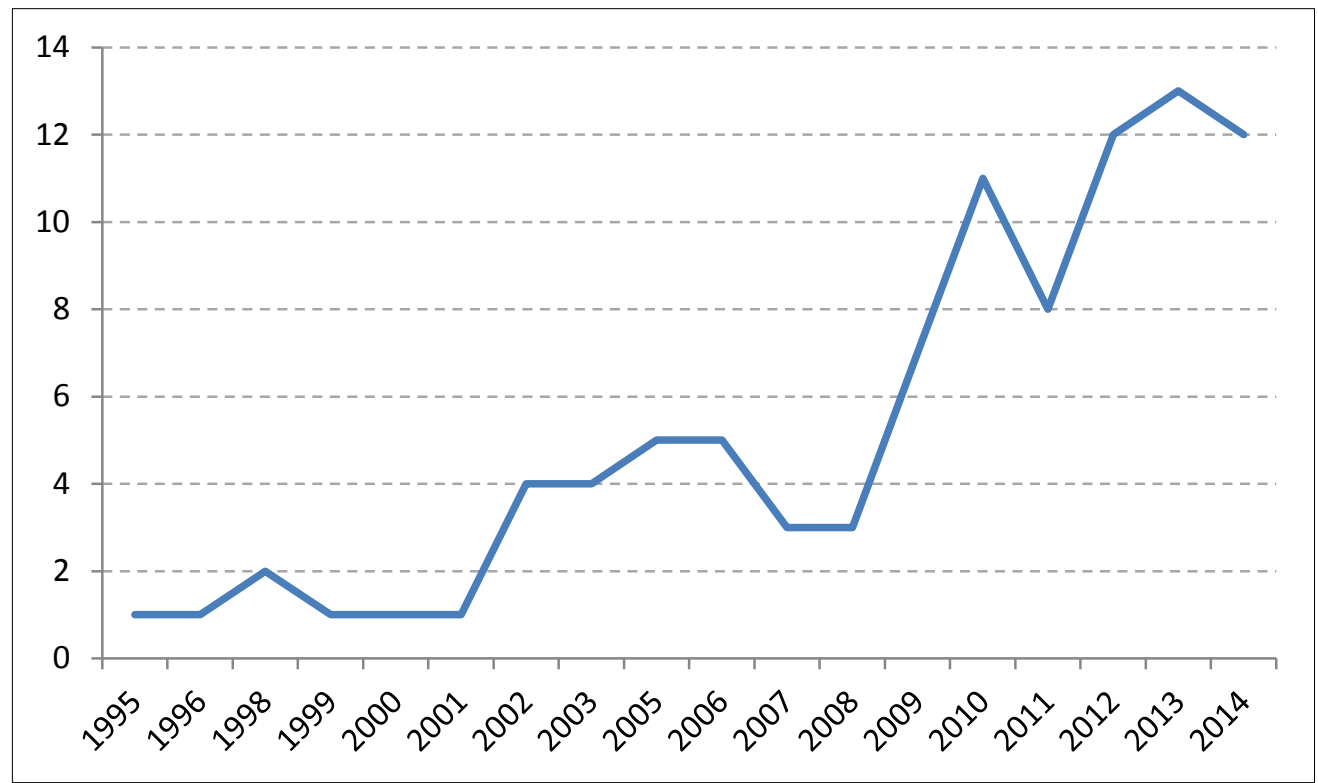

Figura 3- Quantidade de publicações na Scopus onde o termo "sales forecast" é encontrado (Dentre os resultados de "fuzzy")

Fonte: Autores (2015)

As principais referências em previsão de demanda utilizando lógica fuzzy podem ser vistas no Quadro 1 Estes artigos foram destacados, dentre 99 resultados da procura, por serem relativamente novos e por possuírem uma gama bem variada de técnicas aplicadas. Um deles, em especial (Liu et al., 2013), é um Review bem completo, sumarizando conceitualmente as técnicas. 
Quadro 1 - Cinco dos principais trabalhos encontrados

\begin{tabular}{|c|c|c|c|c|}
\hline Autores & Trabalho & Periódico & Métodos & Segmento \\
\hline $\begin{array}{l}\text { Linares-Mustarós, } \\
\text { S.; Merigó, J. M.; } \\
\text { Ferrer-Comalat. J. } \\
\quad \text { C. (2015) }\end{array}$ & $\begin{array}{l}\text { Processing Extreme } \\
\text { Values in Sales } \\
\text { Forecasting }\end{array}$ & $\begin{array}{l}\text { Cybernetics and } \\
\text { Systems }\end{array}$ & Números Fuzzy triangulares & $\begin{array}{l}\text { Previsão de vendas } \\
\text { em novos negócios }\end{array}$ \\
\hline $\begin{array}{l}\text { Xia, M.; Wong, W.K. } \\
\text { (2014) }\end{array}$ & $\begin{array}{l}\text { A seasonal discrete grey } \\
\text { forecasting model for } \\
\text { fashion retailing } \\
\text { Min }\end{array}$ & $\begin{array}{l}\text { Knowledge- } \\
\text { Based Systems } \\
\quad \text { journal }\end{array}$ & $\begin{array}{c}\text { SGDM (sea- } \\
\text { sonal discrete grey model), GM (Grey forecasting } \\
\text { model), AR (Autoregression), ANN (Artificial } \\
\text { Neural Networks), FGR (Fuzzy Grey Regression } \\
\text { Model), Método Tseng e Markov Melhorado }\end{array}$ & Indústria da moda \\
\hline $\begin{array}{l}\text { Lin, C. C.; Lin, C. L.; } \\
\text { Shyu, J. Z. (2014) }\end{array}$ & $\begin{array}{l}\text { Hybrid multi-model } \\
\text { forecasting system: A } \\
\text { case study on display } \\
\text { market }\end{array}$ & $\begin{array}{l}\text { Knowledge- } \\
\text { Based Systems }\end{array}$ & $\begin{array}{c}\text { Compara os métodos Amortecimento } \\
\text { exponencial, ARIMA (Auto Regressive Integrated } \\
\text { Moving Average, BPNN (Back-Propagation } \\
\text { Neural Network, SVR (Support Vector } \\
\text { Regression) e ANFIS (Artificial Neural Network e } \\
\text { Fuzzy Inference Systems). Ao final, um sistema } \\
\text { híbrido integra os modelos. }\end{array}$ & $\begin{array}{l}\text { Produção de } \\
\text { Monitores }\end{array}$ \\
\hline Liu, N. et al. (2013) & $\begin{array}{l}\text { Sales Forecasting for } \\
\text { Fashion Retailing } \\
\text { Service Industry: A } \\
\text { review }\end{array}$ & $\begin{array}{l}\text { Mathematical } \\
\text { problems in } \\
\text { engineering }\end{array}$ & $\begin{array}{c}\text { Review - Comparação conceitual de vários } \\
\text { métodos, entre eles, vários que utilizam Lógica } \\
\text { Fuzzy }\end{array}$ & Indústria da Moda \\
\hline $\begin{array}{l}\text { Du, S. F. et al. } \\
\quad(2013)\end{array}$ & $\begin{array}{l}\text { Demand forecasting of } \\
\text { perishable farm } \\
\text { products using support } \\
\text { vector machine }\end{array}$ & $\begin{array}{l}\text { International } \\
\text { Journal of } \\
\text { Systems Science }\end{array}$ & $\begin{array}{c}\text { Combina SVM (Supporting Vector Machine) com } \\
\text { Teoria Fuzzy }\end{array}$ & $\begin{array}{l}\text { Produtos agrícolas } \\
\text { perecíveis }\end{array}$ \\
\hline
\end{tabular}

Fonte: Elaborado pelos autores

\subsection{O processo de gestão da demanda}

O processo de Previsão de Demanda é um dos processos fundamentais em uma Cadeia de Suprimentos (LAMBERT, 2004). A Figura 4 mostra os principais processos que devem ser considerados na Gestão de uma Cadeia de Suprimentos.

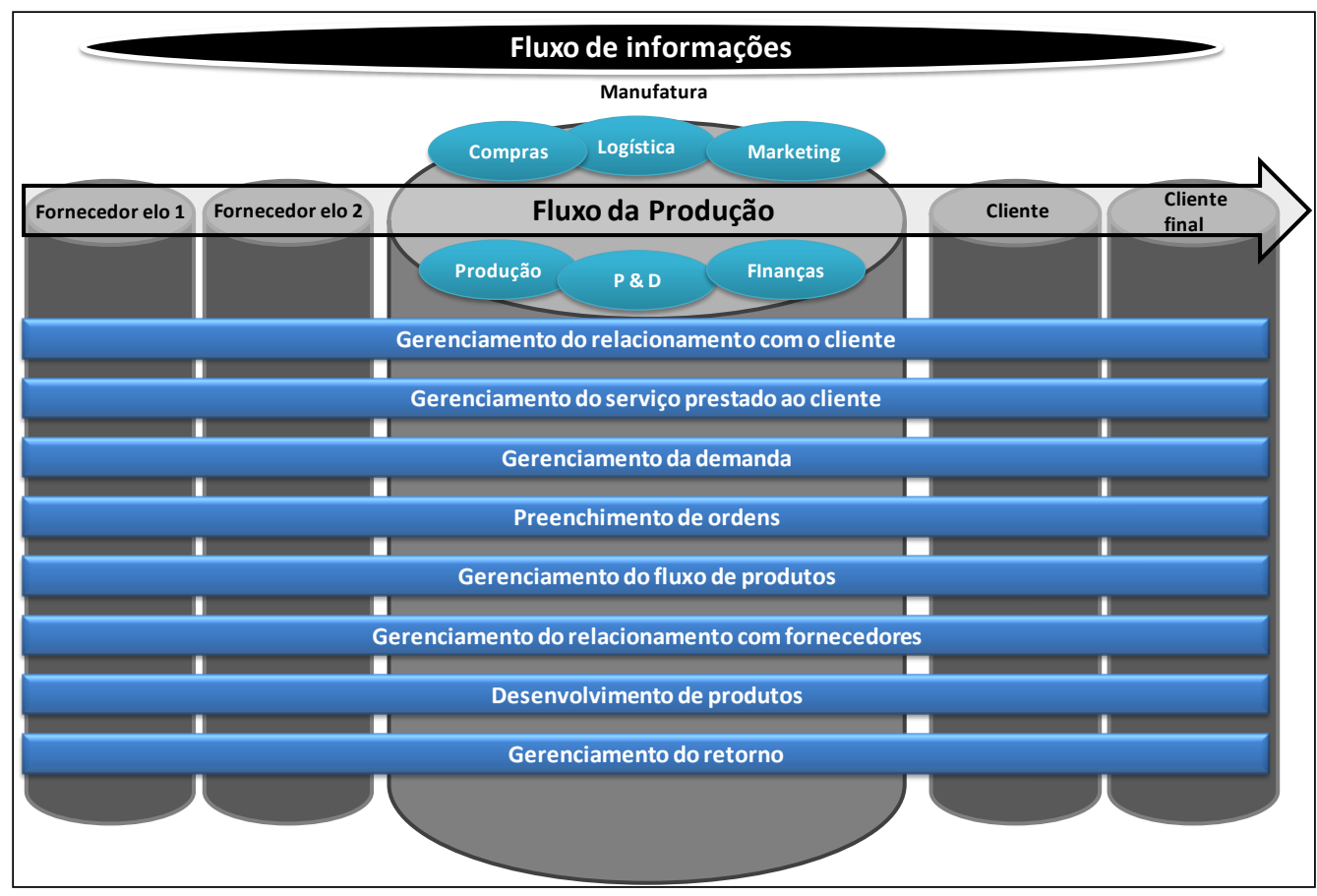

Figura 4 - Processos de Negócios de Cadeias de Suprimentos

Fonte: Lambert, 2004 
O planejamento da demanda tem sido um dos principais aspectos em que os gestores concentram esforços para encontrar uma solução ótima. A questão da previsão de demanda está diretamente relacionada com os estoques e a produção.

No caso de uma produção contra pedido (produção puxada) faz-se necessária uma resposta rápida ao mercado para que a produção tenha tempo de reagir ao pedido colocado. No caso da produção empurrada, de acordo com a previsão de vendas, há a necessidade de se determinar o nível de confiança da previsão de acordo com os SLAs (Service Level Agreements). Com os altos SLAs exigidos pelo cliente, foram desenvolvidos diversos métodos de planejamento da demanda, sejam eles quantitativos (amortecimento exponencial, médias móveis, decomposição clássica, redes neurais, Lógica Fuzzy e etc.), qualitativos (Técnica Delphi) ou ainda iniciativas de planejamento integrado e colaborativo, como, por exemplo, o S\&OP, que basicamente visa a integrar os estoques, a produção e a previsão de demanda.

Ainda para Lambert (2004), o processo de Gerenciamento da Demanda pode ser detalhado de acordo com o mostrado na Figura 5.

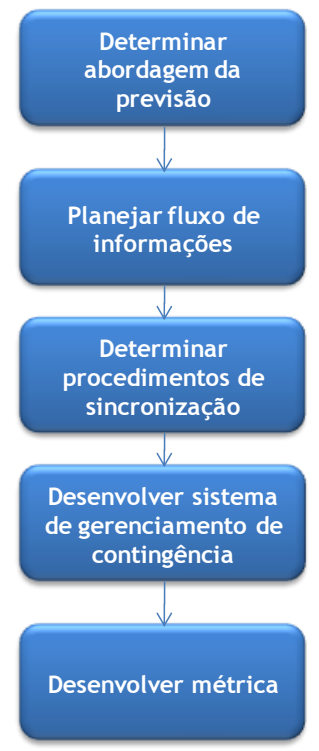

Figura 5 - Gerenciamento da Demanda - Panorama Estratégico

Fonte: Lambert (2004)

Vale ressaltar que, entre os métodos qualitativos, estão às técnicas baseadas no consenso de opiniões, enquanto que os métodos quantitativos se caracterizam pelo emprego de técnicas estatísticas para obtenção das previsões de demanda (LUSTOSA et al., 2008). Para Wanke e Julianelli (2011), apesar dos evidentes benefícios que tais iniciativas de previsão colaborativa de demanda trazem, nem sempre as motivações e desafios a serem enfrentados estão claros para as empresas.

Conclui-se então, que é fundamental conhecer os problemas e impactos nas decisões de áreas funcionais para que se entendam as principais motivações e desafios para a implantação de programas de colaboração entre as empresas. Entre os principais impactos que um planejamento da demanda mal empregado pode ocasionar, pode-se mencionar a falta de produtos, que gera um custo tangível da perda de vendas e um custo intangível de deterioração de marca e perda de clientes. Tais falhas podem ser resultado de alguns fatores cruciais no planejamento da demanda, como:

a) Escolha mal feita do software de previsão de demanda; 
b) Técnicas e formalismo estatísticos não aderentes à realidade da empresa.

\subsection{Previsão de Demanda}

Para iniciar o processo formal de previsão de demanda é necessário definir claramente seu objetivo principal, que está associado à real necessidade de se realizar uma previsão. Dependendo deste objetivo, determinam-se, entre outros aspectos, qual o nível de precisão, quais os prazos e recursos necessários e disponíveis, etc.

A partir da definição dos objetivos e da abrangência do processo de previsão, o passo seguinte consiste na coleta de dados, que inclui dados históricos e informações sobre eventos passados e futuros que podem influenciar o comportamento das vendas de um determinado produto. Uma análise preliminar destes dados pode indicar a necessidade de correção ou mesmo de exclusão de alguns dados históricos. Evidentemente, a quantidade e o tipo das informações estão associados ao propósito da previsão, assim como a natureza do modelo de previsão empregado (LUSTOSA et al., 2008).

\subsection{Conjuntos Fuzzy}

A Teoria de Conjuntos Fuzzy e os Conceitos de Lógica Fuzzy podem ser utilizados para traduzir, em termos matemáticos, a informação imprecisa expressa por um conjunto de regras linguísticas do tipo SE-ENTÃO. Se um ser humano for capaz de articular o seu raciocínio como um conjunto de regras da forma SE-ENTÃO, é possível criar um sistema de inferência, cujo algoritmo pode ser implementado através de um programa computacional, no qual a Teoria de Conjuntos Fuzzy e Lógica Fuzzy fornecem o ferramental matemático para se lidar com as tais regras linguísticas (TANSCHEIT, 2004).

Desenvolvida por Zadeh (1965), a Lógica Fuzzy é a ciência que estuda os princípios formais do raciocínio aproximado e tem como base a Teoria de Conjuntos Fuzzy. A Lógica Fuzzy lida com a imprecisão intrínseca, ou seja, associada com a descrição das propriedades de um fenômeno, e não com a imprecisão associada à medição do fenômeno propriamente dito. Enquanto a Lógica Clássica foi construída com base no princípio de bivalência, a Lógica Fuzzy foi construída com base no princípio da multivalência a partir da adaptação dos conceitos já estabelecidos de Lógica Clássica e da definição de operadores à semelhança dos tradicionalmente utilizados.

Um Sistema de Inferência Fuzzy faz o mapeamento de entradas precisas em saídas precisas utilizando à Lógica Fuzzy, conforme explicitado na Figura . Neste Sistema de Inferência Fuzzy, consideram-se entradas não-fuzzy, ou precisas - resultantes de medições ou observações (conjuntos de dados, por exemplo), que é o caso da grande maioria das aplicações práticas. Em virtude disto, é necessário efetuar-se um mapeamento destes dados precisos para os conjuntos fuzzy (de entrada) relevantes, o que é realizado no estágio de fuzzificação. Neste estágio, ocorre também a ativação das regras relevantes para uma dada situação. Uma vez obtido o conjunto fuzzy de saída através do processo de inferência (modus ponens generalizado), no estágio de defuzzificação é efetuada uma interpretação dessa informação. Isto se faz necessário, pois em aplicações práticas, geralmente são requeridas saídas precisas. 


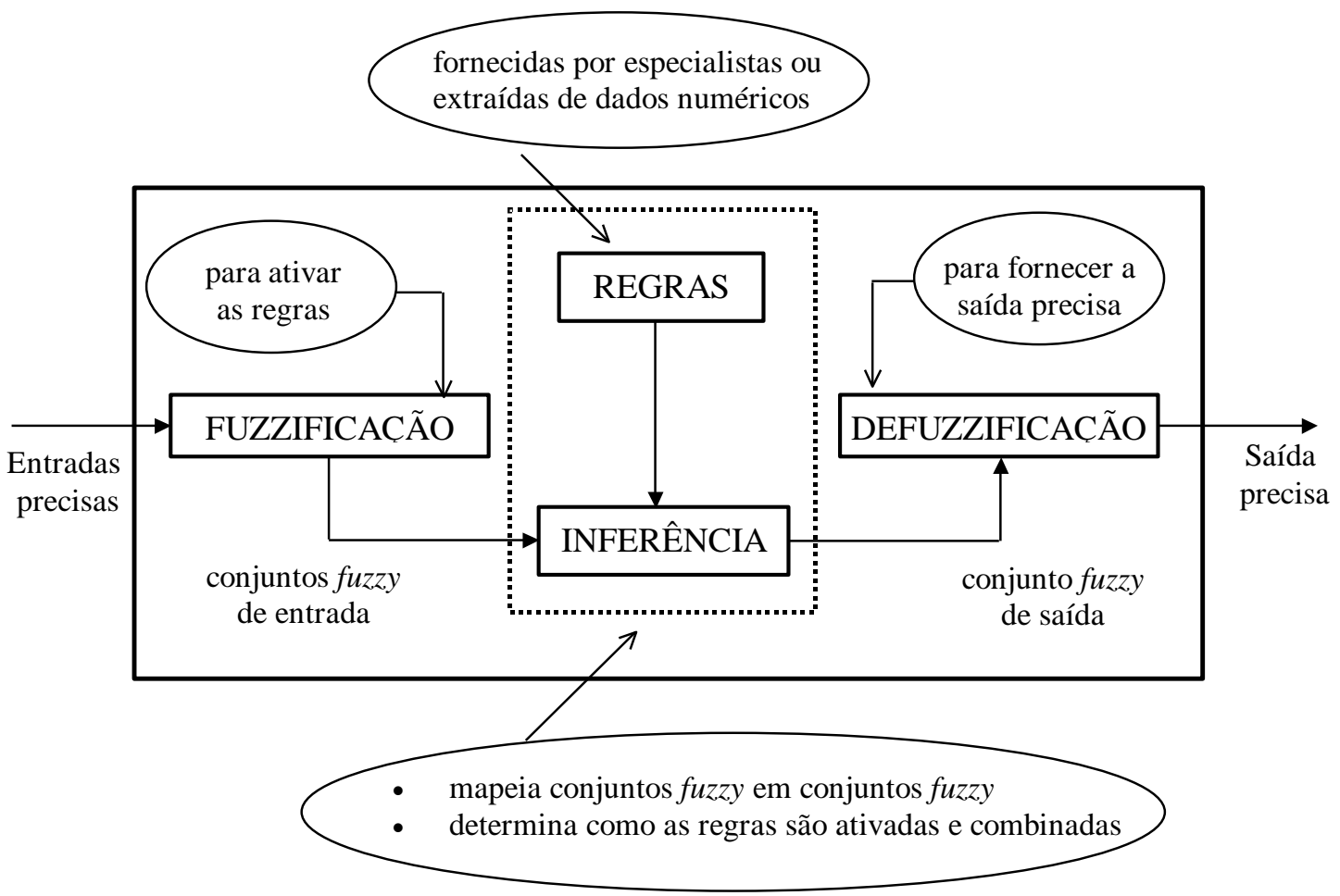

Figura 6 - Sistema de inferência Fuzzy

Fonte: Tanscheit, 2004

\subsection{Técnicas de Previsão de Séries Temporais}

De acordo com Morettin e Toloi (1986), uma série temporal é uma coleção de observações, feitas em diferentes instantes de tempo, não necessariamente igualmente espaçadas e sujeitas a variações aleatórias. Como exemplos, tem-se:

a) Valores mensais de temperatura da cidade de São Paulo.

b) Quantidades anuais de chuva na cidade de Fortaleza.

c) Valores mensais do índice de produto industrial no Brasil.

d) Alturas de marés no porto de Santos, obtidas através de um marégrafo.

e) Registro de um eletrocardiograma (ECG) de uma pessoa.

f) Registro do movimento da crosta terrestre, obtido através de um sismógrafo.

Nos exemplos (a) - (c) têm-se séries temporais discretas, ao passo que (d) - (f) são exemplos de séries temporais contínuas. Na maioria das vezes, as séries discretas são observadas em instantes de tempo igualmente espaçados, como nos casos acima. Mas é possível que as observações apareçam de forma irregular ou, ainda, que haja perda de observações.

A inevitável presença de ruído em sinais experimentais torna o estudo de séries temporais ainda mais difícil, podendo acarretar interpretações errôneas dos resultados. Desta forma, o uso de técnicas adequadas é extremamente importante na análise destas séries (SAVI, 2006). Um modelo adequado que forneça uma visão do funcionamento do mecanismo gerador dos dados pode ser usado, por exemplo, para prever valores futuros da série (FERREIRA, 2008).

Algumas características são particulares deste tipo de dados, como, por exemplo: 
a) É preciso levar em conta a ordem temporal das observações.

b) Fatores complicadores como presença de tendências e variação sazonal ou cíclica podem ser difíceis de estimar ou remover.

c) A seleção de modelos pode ser bastante complexa e as ferramentas podem ser de difícil interpretação.

d) É mais difícil lidar com observações perdidas e dados discrepantes, devido à natureza sequencial.

Apesar de não ser o objetivo do trabalho discutir exaustivamente técnicas de previsão de demanda, cabe uma breve discussão acerca de alguns métodos. Neste trabalho, efetua-se a previsão de demanda por meio da Lógica Fuzzy e, para efeito de comparação, faz-se uso da Decomposição Clássica.

\subsubsection{Decomposição Clássica}

A Decomposição Clássica pode ser classificada como uma técnica de séries temporais de modelo aberto, que analisa em primeiro lugar as séries disponíveis de modo a identificar as componentes que existem e a sua natureza.

Segundo Wanke \& Julianelli (2011), há várias técnicas de modelo aberto (OMTS), entre elas a análise por decomposição, a análise espectral, a análise de Fourier e a ARIMA (Box-Jenkins). Todas estas técnicas, além de analisarem os componentes presentes nas séries, exigem um histórico razoável de dados. De fato, na maioria das técnicas de OMTS recomenda-se um histórico de 48 períodos.

Cada uma das quantidades das parcelas é expressa em unidades de demanda que se somam. No entanto, o modelo multiplicativo é mais utilizado na prática e é representado pela equação 1:

$$
\mathrm{Y}=(\mathrm{T}) \cdot(\mathrm{S}) \cdot(\mathrm{C}) \cdot(i)
$$

onde:

$\mathrm{Y}=$ valor da série (demanda prevista)

$\mathrm{T}=$ componente de tendência

$\mathrm{S}=$ componente de sazonalidade

$\mathrm{C}=$ componente cíclica

$i=$ resíduo devido a flutuações irregulares

\subsubsection{Previsão por Lógica Fuzzy}

A previsão por meio da Lógica Fuzzy é realizada a partir do método de extração de regras de Wang \& Mendel (1992), que é apresentado de forma resumida a seguir.

Seja $x(k), k=1,2, \ldots$, uma série temporal. Supõe-se como objetivo, dada uma janela de $n$ medidas $x(k-n+1), x(k-n+2), \ldots, x(k)$, determinar $x(k+l)-o$ valor de $x$ em $l$ pontos à frente $(n$ e $l$ : inteiros positivos), como mostra a Figura 7. 


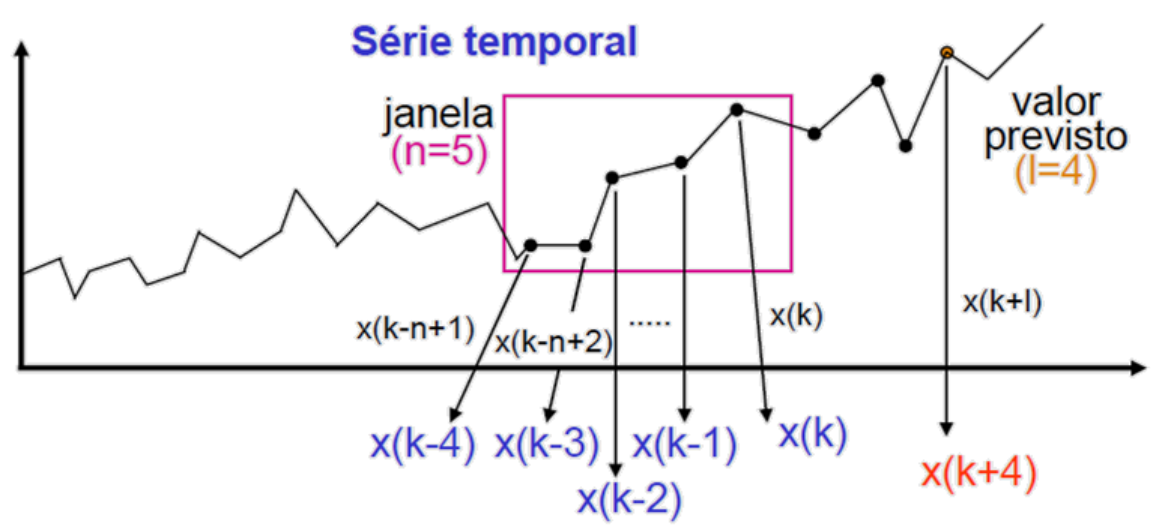

Figura 7 - Extração de regras

Fonte: Tanscheit, 2011

Para extrair as regras são seguidos alguns passos:

a) Especificam-se previamente os conjuntos fuzzy e associam-se os dados a estes conjuntos.

b) Como o valor a ser previsto depende de $n$ valores passados de $x$, cada regra possui $n$ antecedentes.

Primeiramente, divide-se a série, contida nos limites $\left[\mathrm{U}^{-}, \mathrm{U}^{+}\right]$, em um número ímpar de conjuntos fuzzy. A Figura mostra um exemplo com 7 conjuntos, representados por funções de pertinência triangulares.
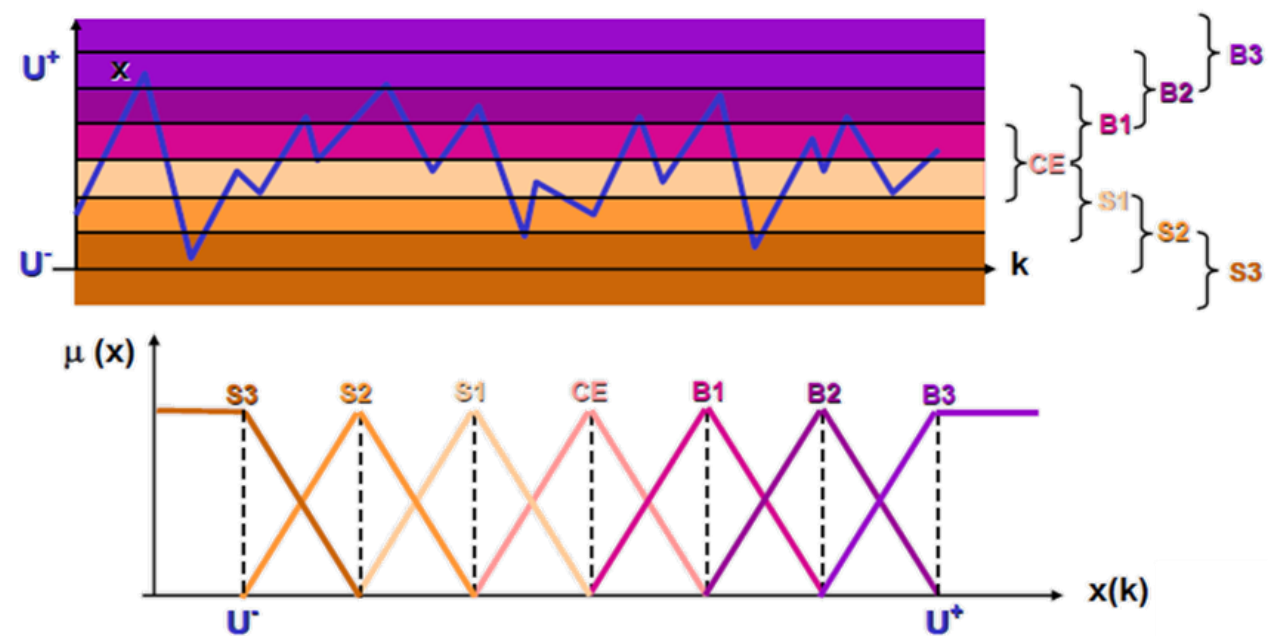

Figura 8 - Formação de regras

Fonte: Tanscheit, 2011

Para gerar as regras deve-se:

a) Determinar o tamanho da janela $n$.

b) Determinar o horizonte de previsão.

Executar os passos a seguir, para cada regra $j$. 
c) Determinam-se os graus de pertinência dos elementos $x_{j}$.

d) Atribui-se a cada variável o conjunto com maior grau.

e) Obtém-se uma regra para cada par entrada-saída.

O tamanho da janela $n$ depende da aplicação e de quantos e quais valores passados têm mais influência no futuro. O horizonte de previsão é definido em função de quantos valores a frente se deseja fazer a previsão

\subsubsection{Indicador de qualidade do método de previsão}

Os dois métodos foram aplicados e os resultados foram analisados e comparados de acordo com critérios de desempenho baseado em medidas de erro, isto é, os desvios entre valores previstos e efetivamente observados. O método utilizado foi o MAPE (Mean Absolute Percentage error), expresso na equação 2. O MAPE é uma medida da acurácia da previsão e, para calculá-lo, considera-se desvios relativos ou percentuais no cálculo do erro. A partir da comparação dos resultados pôde-se eleger o método de previsão mais eficiente para esta aplicação.

$$
M A P E=\frac{\sum_{t=1}^{n}\left|\frac{D_{t}-F_{t}}{D_{t}}\right|}{n}
$$

$D_{t}=$ Valor real de demanda no período $t$

$F_{t}=$ Valor previsto de demanda no período $t$

$n=$ Número de períodos.

\section{ANÁLISE DOS RESULTADOS}

A série histórica estudada possui 103 dados ao todo; é mensal, diz respeito a materiais têxteis e foi retirada do site do sistema FIRJAN. O indicador industrial - adimensional - avalia cinco índices de desempenho de 170 indústrias do estado (FIRJAN, 2011). Os primeiros 78 dados foram utilizados para calibrar ambos os métodos. A previsão foi efetuada considerando-se os 25 valores finais. Pela Decomposição Clássica são obtidos os resultados apresentados na Figura 9: 


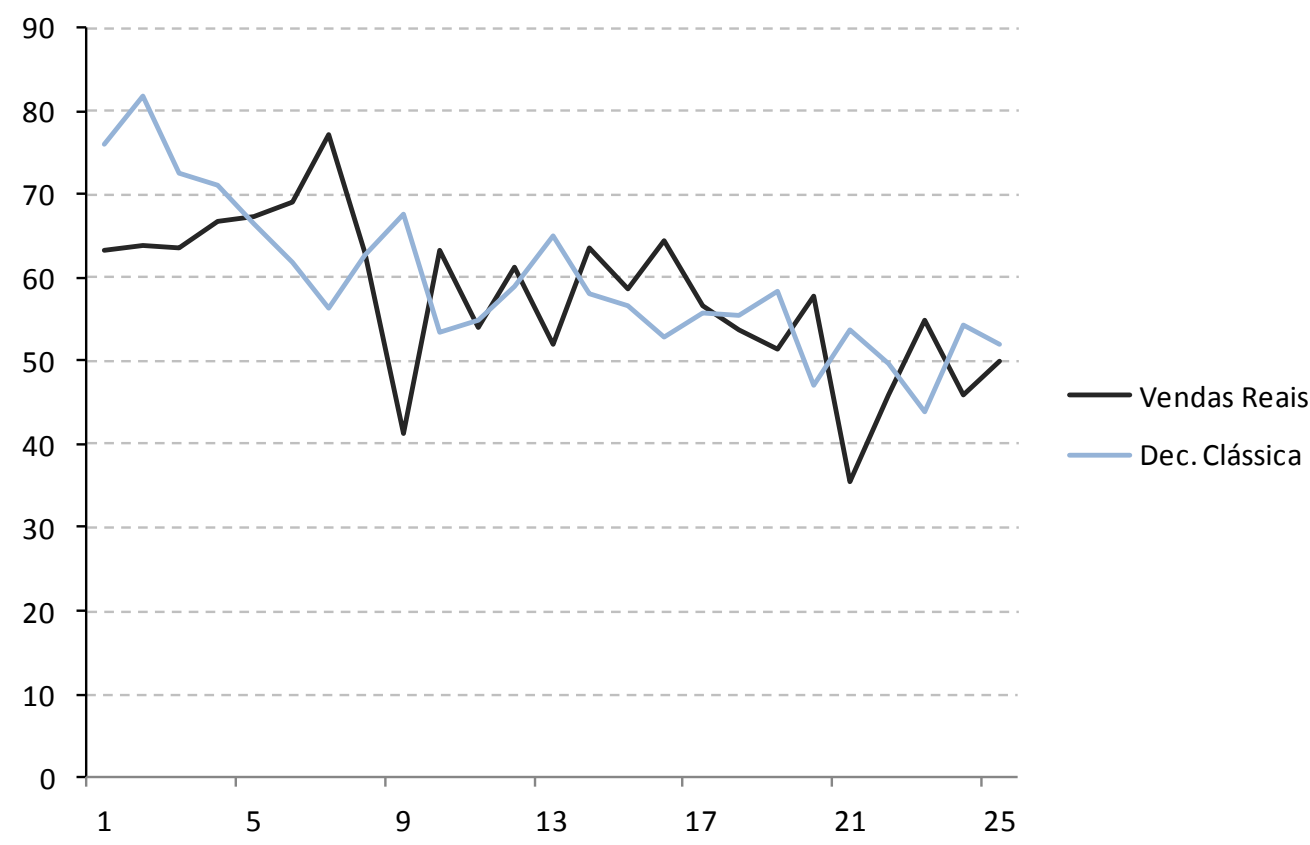

Figura 9 - Decomposição Clássica e vendas Reais

Fonte: Elaborado pelos autores (2015)

O MAPE encontrado é de $15,5 \%$. Para a previsão por meio do método de Wang \& Mendel (1992), os resultados dependem do número de conjuntos fuzzy escolhido. A janela de previsão (valores históricos considerados) é de tamanho 4. Como pode ser observado a partir dos gráficos, o melhor resultado é obtido para 7 conjuntos fuzzy.

Tabela 1 - Resumo dos resultados

\begin{tabular}{lr} 
Técnica & MAPE \\
\hline \hline Fuzzy 7 & $8,40 \%$ \\
Dec. Clássica & $15,50 \%$ \\
Fuzzy 5 & $34,20 \%$ \\
Fuzzy 11 & $35,50 \%$ \\
Fuzzy 9 & $44,10 \%$
\end{tabular}

Fonte: Elaborado pelos autores (2015)

Com alguns ajustes finos é possível obter soluções melhores. Em uma segunda bateria de experimentos, toma-se o melhor resultado $(8,40 \%$ de erro médio percentual) e altera-se o tamanho da janela de previsão. Os resultados são apresentados na Tabela 2:

Tabela 2 - Melhoria do melhor resultado variando a janela

\begin{tabular}{lr} 
Técnica F7 & MAPE \\
\hline \hline Janela 4 & $8,4 \%$ \\
Janela 5 & $8,7 \%$ \\
Janela 6 & $7,7 \%$ \\
Janela 7 & $31,2 \%$
\end{tabular}

Fonte: Elaborado pelos autores (2015)

O melhor MAPE encontrado foi obtido para a janela de tamanho 6. 
As influências do operador usado a para interseção dos antecedentes das regras (mínimo ou produto) e do método de defuzzificação (altura limite ou centroide).

Tabela 3- Variação de parâmetros para melhoria do resultado

\begin{tabular}{lllr} 
Interseção & Implicação & Defuzificação & MAPE \\
\hline \hline Produto & Mínimo & Altura Lim. & $6,6 \%$ \\
Produto & Produto & Altura Lim. & $6,6 \%$ \\
Produto & Produto & Centróide & $6,7 \%$
\end{tabular}

Fonte: Elaborado pelos autores (2015)

O gráfico da Figura apresenta uma comparação entre os melhores resultados.

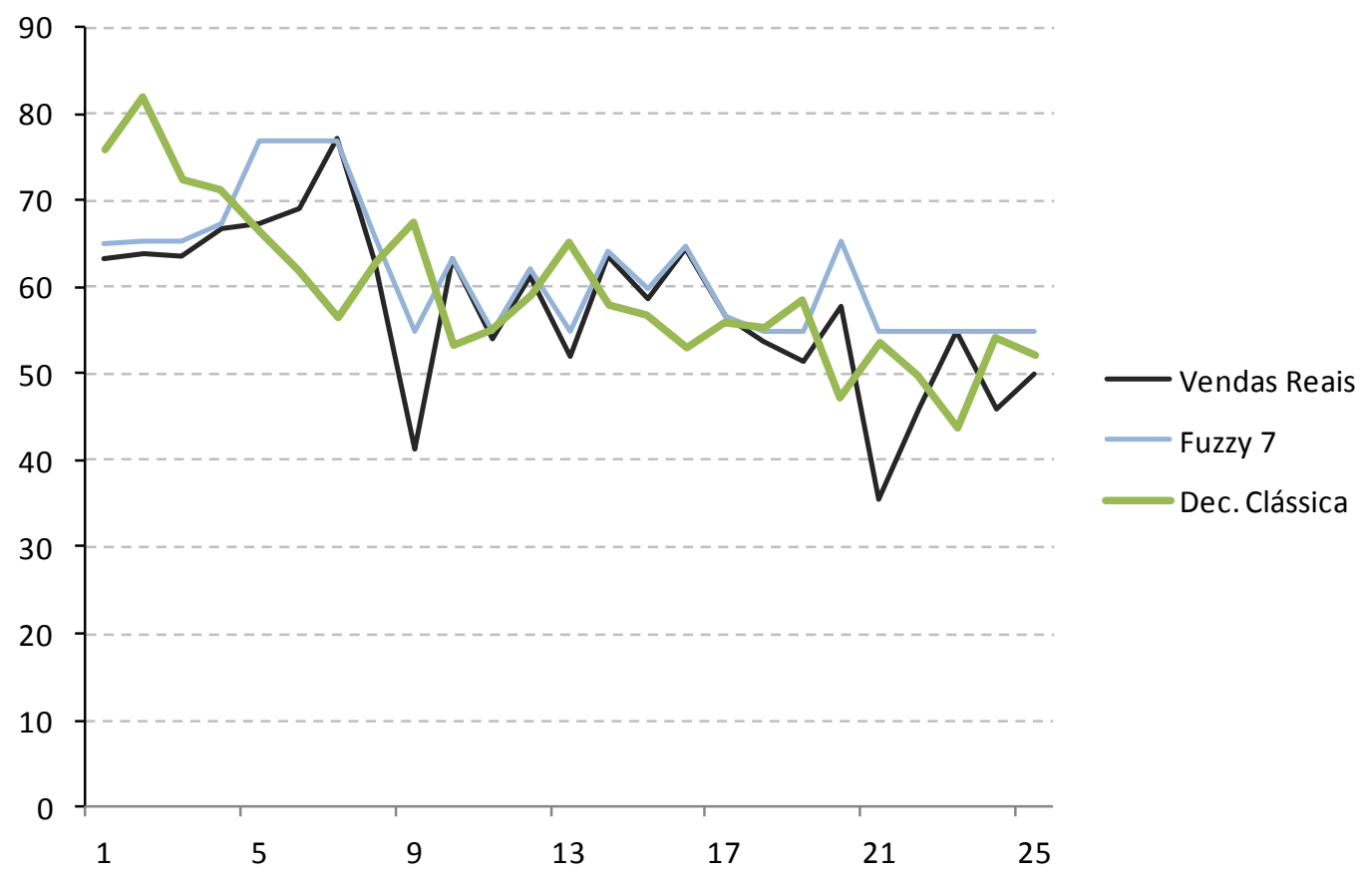

Figura 10 - Comparação das técnicas

Fonte: Elaborado pelos autores (2015)

O MAPE obtido para a previsão por Lógica Fuzzy foi de $6,6 \%$, enquanto que o da Decomposição Clássica foi de $15,5 \%$. Ainda, a análise gráfica mostra que a Decomposição Clássica detecta os vales e picos de demanda com um período de defasagem, o que não ocorre com a Lógica Fuzzy.

\section{CONCLUSÕES}

A Decomposição Clássica é uma técnica bastante difundida e de fácil aplicação. Esta técnica costuma apresentar bons resultados e é indicada para apoiar processos de previsão de demanda, que, em muitas empresas são realizados de forma estritamente qualitativa. Além disso, muitas vezes a meta de vendas da empresa é transformada na previsão, o que é um equívoco (AROZO, 2000). A previsão por Lógica Fuzzy para esta série temporal em especial apresenta melhores resultados, pois é mais flexível do que a decomposição clássica por permitir alterações no número de termos 
linguísticos. Neste trabalho o indicador utilizado para mensurar este erro foi o MAPE, por ser o mais empregado na literatura.

Cabe ressaltar que a decomposição clássica também pode ser melhorada em suas etapas prévias. Esta melhoria pode ser conseguida utilizando-se outro tipo de regressão no cálculo da tendência e combinando métodos qualitativos e quantitativos para a definição das sazonalidades. No entanto, a modificação deste método não é algo trivial e exigiria uma mudança de paradigma por parte da maioria das empresas, que são condicionadas ao uso do Excel e raramente procuram soluções alternativas.

Ainda é possível apontar a possibilidade de alguns estudos futuros, como, por exemplo, verificar como utilizar a Lógica Fuzzy para previsão de demanda sem o uso de séries históricas, mas utilizando variáveis causais como preço e propaganda. Outras técnicas poderiam ser utilizadas tais como amortecimentos exponenciais e Redes Neurais Artificiais.

\section{REFERENCIAS}

AROZO, R. Sales and Operations Planning: Uma maneira de obter ganhos com integração interna. COPPEAD, 2000 .

DU, X. F., LEUNG, S. C. H., ZHANG, J. L., LAI, K.K. Demand forecasting of perishable farm products using support vector machine. International journal of systems Science. v. 44, n. 3, p. 556-567, mar. 2013.

FERREIRA, B. B. Aplicação de Ferramentas de Lógica Nebulosa à Predição de Séries Temporais. Rio de Janeiro: Instituto Militar de Engenharia, Curso de Mestrado em Engenharia Mecânica, Dissertação, 2008. 124 p.

FIRJAN. Série de dados utilizados na previsão. Disponível em: <http://www.firjan.org.br/data/pages/4028808120E98EC7012122BA8A14346E.htm>. Acesso em: 2011.

LAMBERT, D. M. Supply Chain Management: Processes, Partnerships, Performance. 2. ed. Florida, SCMI, 2004. LIN, C.C., LIN, C.L., SHYU, J. Z. Hybrid multi-model forecasting system: a case study on display market. Knowledgebased systems. v. 71, p. 279-289, nov. 2014.

LIU, N., REN, S., CHOI, T. M., HUI, C. L., NG, S. F. Sales Forecasting for Fashion Retailing Service Industry: A Review. Mathematical Problems in Engineering. v. 2013, Article ID 738675, 9 p., 2013. doi:10.1155/2013/738675

LINARES-MUTARÓS, S. MERINGÓ, J. M. FERRER-COMELAT, J. C. Processing extreme values in sales forecasting. Cybernetics and Systems. v. 46, n. 3-4, p. 207-229, mai. 2015.

LUSTOSA, L. J.; MESQUITA, M. A.; GONÇAlVES, O. L.; OLIVEIRA, R. J. Planejamento e Controle da Produção. 1. ed. Rio de Janeiro: Elsevier, 2008.

MORETTIN, P. A.; TOLOI, C. M. Séries Temporais. São Paulo: Atual, 1986.

SAVI, M. A. Dinâmica Não-Linear e Caos. 1. ed. Rio de Janeiro: E-papers Serviços Editoriais Ltda, 2006.

TANSCHEIT, R. Sistemas Fuzzy, Departamento de Engenharia Elétrica, Pontifícia Universidade Católica do Rio de Janeiro, Rio de Janeiro. 2004.

WANKE, P.; JULIANELLI, L. Previsão de vendas: processos organizacionais e métodos quantitativos e qualitativos. 2. ed. São Paulo: Atlas, 2011.

WANG, L. X., MENDEL, J. M. Generating Fuzzy Rules by Learning from Examples, IEEE Trans. On Systems, Man and Cybernetics, , V 22, n. 6, p.1414-1427 1992.

XIA, M. WONG, W. K. A seasonal discrete grey forecasting model for fashion retailing. Journal knowledge-based systems. V. 57, p. 119-126, Fev. 2014.

YIN, R. Estudos de Caso: Planejamento e Métodos. 2. ed. Porto Alegre: Bookman, 2010.

ZADEH, L. "Fuzzy Sets", Information and Control, vol. 8, p. 338-353, 1965. 